\title{
Antropología
}

\section{La percepción del envejecer en una población de ancianos}

\section{The perception of the grow older in a elderly population}

\section{A percepção do envelhecer em uma população de idosos}

Eliane Santos Cavalcante ${ }^{1}$, Izaura Luzia Silvério Freire ${ }^{2}$, Andréa Tayse de Lima Gomes ${ }^{3}$,

Micheline da Fonseca Silva ${ }^{4}$, Francisco Arnoldo Nunes de Miranda ${ }^{5}$, Gilson de Vasconcelos Torres ${ }^{6}$

${ }^{1}$ Enfermeira. Doutora em Enfermagem. Professora da Escola de Saúde da Universidade Federal do Rio Grande do Norte (UFRN). ${ }^{2}$ Enfermeira. Doutora em Enfermagem. Professora da Escola de Saúde da Universidade Federal do Rio Grande do Norte (UFRN). ${ }^{3}$ Enfermeira. Mestranda do programa de pós-graduação em enfermagem da Universidade Federal do Rio Grande do Norte (UFRN). ${ }^{4}$ Enfermeira. Mestranda do programa de pós-graduação em enfermagem da Universidade Federal do Rio Grande do Norte (UFRN).

${ }^{5}$ Enfermeiro. Doutor em Enfermagem. Professor adjunto da graduação e pós-graduação em Enfermagem da Universidade Federal do Rio Grande do Norte (UFRN).

${ }^{6}$ Enfermeiro. Pós-doutor em Enfermagem. Professor titular da graduação e pós-graduação em Enfermagem da Universidade Federal do Rio Grande do Norte (UFRN).

Cómo citar este artículo en edición digital: Cavalcante, E.S., Freire, I. L. S., Gomes, A.T.L., Fonseca, M.F., Miranda, F.A N., Torres, G.V.(2017). La percepción del envejecer en una población de ancianos. Cultura de los Cuidados (Edición digital) 21,47. Recuperado de http://dx.doi.org/10.7184/cuid.2017.47.12

Correspondencia: Campus universitário, Avenida Senador Salgado Filho, s/n, Lagoa Nova, Natal/RN/Brasil. CEP: 59078-970. Correo electrónico: elianeufrn@hotmail.com Recibido: 07/07/2016; Aceptado: 20/12/2016

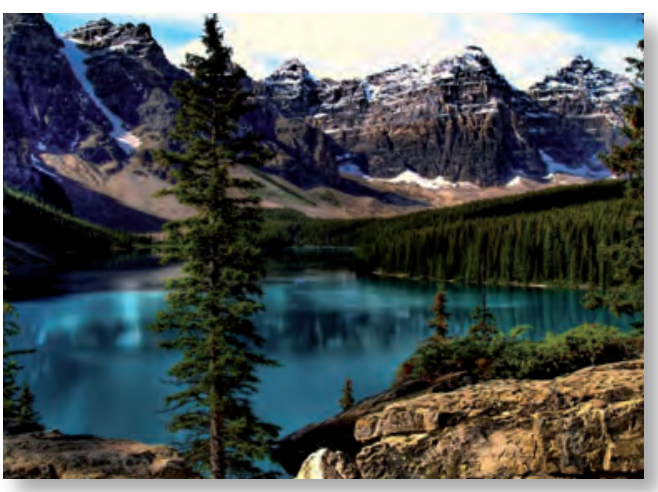

\section{ABSTRACT}

This study aimed to understand the meaning attributed to the aging process by the elderly of a basic health unit. Qualitative, analytical and cross-sectional study, realized in a charity house in the Metropolitan region of the Rio Grande do Norte / Brazil. The sample was composed by 35 elderly and the data collection was between August and December
2014 through semi-structured interview and analyzed according to the Bardin method. Elderly reported that aging is characterized as a burden, moment of solitude and predisposition to disease. Other researched have revealed contentment and welfare notable and hope as to respective epoch lived, acknowledgment for the privilege to reach the advanced age. It is concluded that to improve the quality of life of older people, it is necessary to promote integration between them and the permanency institution as a way to encourage coexistence and social interaction.

Keywords: Aging, aged, health services for the aged, primary health care.

\section{RESUMEN}

Este estudio tuvo como objetivo comprender el significado que se atribuye al proceso de envejecimiento por ancianos de una unidad 
básica de salud. estudio cualitativo, analítico y transversal, realizado en una casa de caridad de la Región metropolitana de Rio Grande do Norte / Brasil. La muestra estuvo compuesta por 35 ancianos y la recolección de datos fue entre agosto y diciembre de 2014 por medio de entrevistas semiestructuradas y analizadas como el método de Bardin. Los ancianos informaron que la vejez se caracteriza como carga, momento de soledad y predisposición a las enfermedades. Otros investigados revelaron certa alegría, bienestar notable y esperanza com respecto al tiempo vivido, agradecimiento por el privilegio de llegar a la edad avanzada. Se concluye que para mejorar la calidad de vida de los ancianos, es necesario promover la integración entre ellos y la institución de permanencia como forma de estimular la convivencia y la interacción social.

Palabras clave: Envejecimiento, anciano, servicios de salud para ancianos, atención primaria de salud.

\section{RESUMO}

Objetivou-se compreender o significado atribuído ao processo de envelhecimento pelos idosos de uma unidade básica de saúde. Estudo qualitativo, analítico e seccional, realizado em uma casa de caridade da região Metropolitana do Rio Grande do Norte/Brasil. A amostra foi composta por 35 idosos e a coleta de dados foi entre agosto e dezembro de 2014 por meio de entrevista semiestruturada e analisadas conforme o método de Bardin. Idosos relataram que a velhice caracteriza-se como fardo, momento de solidão e predisposição a doenças. Outros pesquisados revelaram contentamento e notável bem-estar e esperança a respectiva época vivida, agradecimento pelo privilégio de chegar à idade avançada. Conclui-se que para melhorar a qualidade de vida dos idosos, é necessário promover a integração entre eles e a instituição de permanência como forma de estimular a convivência e interação social.

Palavras-chave: Envelhecimento, idoso, serviços de saúde para idosos, atenção primária à saúde.

\section{INTRODUÇÃO}

A Organização Mundial de Saúde (OMS), por meio das Leis $8842 / 94$ e $10741 / 03$, considera idosa a pessoa com 60 anos ou mais. Apesar de muitas vezes predominar o preconceito e a discriminação contra esse grupo populacional, vem aumentando a consciência de que os idosos podem dar contribuição fundamental à construção de uma sociedade mais humana (Keinert \& Rosa, 2009).

Do ponto de vista biológico, entende-se o envelhecimento como processo dinâmico e progressivo com alterações morfológicas, bioquímicas, funcionais, comportamentais, cognitivos e sociais ocorrendo, assim, interação entre esses fatores que orientam tanto o funcionamento típico quanto atípico do processo do envelhecimento (Combinato et al., 2010).

Estas alterações são dependentes de fatores como estilo de vida, condições socioeconômicas e doenças crônicas. Além do conceito "biológico", considera-se o psíquico, que consiste na relação das dimensões cognitivas e psicoafetivas, as quais interferem na personalidade e no afeto (Fechine, \& Trompiere, 2012). Desse modo, denota-se que a interação entre a biologia, o psicológico, o estilo de vida e as condições socioeconômicas interferem substancialmente nas condições do processo de envelhecimento do indivíduo.

O processo de envelhecimento da população consiste em fenômeno que vem ocorrendo em nível mundial e de forma distinta entre os diversos países do mundo (Rocha, Carval- 
ho, Figueiredo, \& Caldas, 2011; Banza \& José 2012). Segundo a OMS, no ano de 2050, as estimativas para a população de mais de 60 anos é em torno de dois bilhões de pessoas idosas no mundo e a maioria delas vivendo em países em desenvolvimento. A rapidez com que ocorre o envelhecimento populacional, especialmente nos países em desenvolvimento, tornou-se tema da atualidade (Jobin, Sousa, \& Cabrera, 2010).

No Brasil, há previsão de que entre o período de 1970 a 2025 o crescimento do número de idosos seja de $223 \%$ de pessoas com 60 anos ou mais de idade, projetando-se para 2025 um número de 1,2 bilhões de pessoas nesta faixa etária. Esta transição demográfica decorre de diminuições importantes de taxas de natalidade, fecundidade e coeficientes de mortalidade, que geraram aumento da expectativa de vida ao nascer (Fabrício \& Rodrigues, 2008).

Desse modo, o processo do envelhecimento traz consigo algumas peculiaridades como mudança nos padrões de saúde, capacidade funcional, independência financeira e a possibilidade de vivenciar debilidades que torne necessário residir com familiares. Estudos mostram que $43 \%$ dos idosos não institucionalizados acima de 85 anos de idade necessitam de assistência nas atividades básicas de vida diária que são, geralmente, providas por seus familiares (Torres et al., 2009).

Observa-se, portanto, um organismo fragilizado pelo processo do envelhecimento, no entanto, a Qualidade de Vida (QV) do idoso se determina, principalmente, pela garantia de autonomia e decisão sobre si e sua vida. Acredita-se assim, que para o cuidado ao idoso é necessário revelar e interpretar fatores ambientais, sociais e econômicos, e por meio dessas interfaces viabilizarem um cuidado integral a essa clientela. Nesse sentido, o conceito para QV se torna subjetivo, multidimensional e influenciado, de acordo com o significado de cada uma dessas dimensões para o sujeito (Moliterno, Faller, Borghi, Marcon, \& Carreira, 2012).

A OMS define QV como: “[...] a percepção que o indivíduo tem de sua posição na vida dentro do contexto de sua cultura e do sistema de valores de onde vive, e em relação a seus objetivos, expectativas, padrões e preocupações" (Tavares, Araújo, \& Dias, 2011).

Nesse ínterim, reconhece-se que várias são as circunstâncias vivenciadas de forma distinta pelas pessoas idosas, tais como as perdas ou não, cognitivas, físicas e mentais e as mudanças ou não na personalidade, na vida social e produtiva, que afetam de algum modo a autonomia e a independência para o viver diário e a qualidade de vida (Gonçalves et al., 2013).

A velhice, para ser compreendida, há que ser considerada em sua multidimensionalidade, onde convivem mudanças cognitivas, físicas, de papéis sociais e familiares, de perdas e ganhos, no ambiente sociocultural onde ela é construída e vivida (Almeida, Mochel, \& Oliveira, 2011).

Com isso, a preocupação com esta parcela da população vem aumentando, pois os idosos apresentam condições específicas que os tornam mais vulneráveis a perdas tanto do ponto de vista funcional como emocional, econômico e social, predispondo-os, principalmente, à presença de várias doenças, baixa autoestima, depressão, incapacidade para realizações e pobreza, com grandes interferências na QV (Marin, Cecílio, Rodrigues, Ricci, \& Druzian, 2008).

Dentro desse contexto fica nítido que o envelhecimento populacional é notoriamente um fato demográfico relevante com importantes implicações para a saúde física e mental, 
para a QV e para programas de saúde pública (Floriano \& Dalgalarrondo, 2007).

Assim, o idoso tem merecido atenção especial, pois o processo de envelhecer saudável implica cuidados de promoção, prevenção, educação e intervenção e nesse contexto requer envolvimento e qualificação dos profissionais da atenção básica, como as Estratégias de Saúde da Família (ESF), com abordagem multiprofissional e interdisciplinar (Rocha et al., 2011).

Infere-se, então, que para ocorrer efetiva adequação dos serviços de saúde no atendimento a pessoa idosa, é importante que os profissionais da saúde, em especial o enfermeiro, esteja qualificado para compreender as especificidades de saúde da pessoa idosa, assistida tanto pela ESF, quanto por níveis mais complexos de atenção (Queiroz, Fernandes, Cirino, Oliveira, \& Menezes, 2010).

Estratégias de capacitação dos profissionais de saúde se tornam essenciais para a realização do cuidado, visando à promoção, prevenção e a reabilitação. Essa realidade evidencia importante e necessária reflexão sobre os serviços prestados pelos trabalhadores de saúde na busca pela qualidade do atendimento (Andrade et al., 2011).

$\mathrm{Na}$ atualidade existem programas específicos de educação em saúde que visa o bem-estar dos idosos, por meio de redes assistenciais com modernização nos seus serviços, como a Política Nacional do Idoso (PNI), o Pacto em Defesa da Vida por tratar-se de conjunto de compromissos, em que a saúde da pessoa idosa destaca-se como uma das prioridades pactuadas (Teixeira \& Neri, 2008).

Diante da transição demográfica, resultando no aumento da população idosa no Brasil e no mundo, este trabalho objetivou compreender o significado atribuído ao processo de en- velhecimento pelos idosos de uma unidade básica de saúde.

\section{METODOLOGIA}

Estudo do tipo exploratório, descritivo, analítico e seccional, com abordagem qualitativa, realizado em uma casa de caridade localizada na região Metropolitana do Rio Grande do Norte/Brasil, onde há 60 idosos institucionalizados.

Os critérios de inclusão foram: idade a partir de 60 anos, lucidez, boa audição e dicção, participação voluntária e assinatura do Termo de Consentimento Livre e Esclarecido (TCLE). Com base nos critérios de elegibilidade, foram selecionados 35 idosos para a pesquisa, de ambos os sexos e com idades entre 60 e 85 anos. Os dados foram coletados no período de agosto a dezembro de 2014, por meio de uma entrevista utilizando um instrumento com questões semiestruturadas, realizada de forma individual.

Ressalta-se que os participantes do estudo foram identificados por números arábicos (Idoso 1, Idoso 2... Idoso 34) a fim de preservar o anonimato dos mesmos.

Posteriormente, as entrevistas foram transcritas e analisadas de acordo com análise categorial de conteúdo de Bardin, que consta de conjunto de técnicas de análise das comunicações, visando obter, por procedimentos objetivos e sistemáticos de descrição do conteúdo das mensagens, indicadores (quantitativos ou não) que permitam a inferência de conhecimentos relativos às condições de produção/ recepção destas mensagens (Oliveira, 2008).

A execução dessa pesquisa seguiu em conformidade com os parâmetros éticos e legais que regem a Resolução 466/12 do Conselho Nacional de Saúde. Para tanto, o projeto de pesquisa foi previamente submetido ao Comi- 
tê de Ética em Pesquisa da Universidade Federal do Rio Grande do Norte (CEP/UFRN), o qual, após apreciação ética, obteve parecer favorável com parecer de número 562.318 e Certificado de Apresentação para Apreciação Ética 21996313.7.0000.5537.

\section{RESULTADOS}

Nesse estudo dividiu-se a percepção do processo de envelhecimento da população pesquisada em dois blocos: "O envelhecer como desgaste natural" e "A velhice enfrentada como o fim da vida”.

\section{O envelhecer como desgaste natural}

Nos discursos analisados, percebe-se que o processo de envelhecimento ainda se encontra arraigado no ciclo vital: nascer, crescer e morrer, ou seja, o envelhecimento faz parte da vida cotidiana do individuo, isto pode ser observado nas falas dos idosos 21 e 19.

É o que Deus deixou pra natureza humana, nascer, crescer e morrer (Idoso 21).

Tem que ficar mesmo, é aceitar, se tiver com saúde é bom (Idoso 19).

Percebe-se também, contentamento e emoção nas falas dos pesquisados, além de uma notável associação de bem-estar e esperança a respectiva época vivida, agradecimento pelo privilégio de chegar à idade avançada. Mesmo que algum desvio de saúde estivesse no corpo consideraram que esta é a última e mais rica etapa da vida.

É o passar dos tempos, já ter vencido muita coisa e ter esperança de vencer mais (Idoso 4).

É agradecer os anos que já vivi (Idoso 34).

$\mathrm{O}$ pensamento de aceitar a velhice como um bem e um sinal de experiência e sabedoria é compartilhado com os nossos entrevistados e pode ser exemplificado nos seguintes discursos:
É bom, sinal que viveu muito aprendeu mais coisas (Idoso 6).

É melhor do que quando eu era nova, depois de velha estou no meu querer (Idoso 14).

\section{A velhice enfrentada como o fim da vida}

As falas dos Idosos 5 e 13 denotam a percepção da velhice como a fase mais difícil da vida, verdadeiro fardo, momento de solidão e desengano.

Idade, doença, queria ser nova, eu era felize num sabia (Idoso 5).

A velhice traz muitas decepções, vivo de lembranças do passado. Viver é solidão (Idoso 13).

Além disso, observou-se entre as falas dos idosos a desesperança em relação a esta etapa do ciclo vital, a perda da expectativa de vida, maior fragilidade no enfrentamento das dificuldades e para essas pessoas a velhice tornouse algo com conotação negativa.

É num prestar mais, perder o gosto de muita coisa (Idoso 22).

É ficar como eu, doente, sem poder fazer nada, num posso trabalhar, num posso andar, eu nova fazia tudo, hoje tenho vontade e num tenho mais condição (Idoso 25).

Outro ponto relevante foi que os participantes representaram a velhice como a perda do papel social de trabalhador. Aqui a velhice significa momento de declínio da capacidade para trabalhar, enfatizada pela perda da utilidade social da pessoa idosa.

Infelizmente o tempo passou e eu nem vi, envelhecer é não poder mais fazer nada (Idoso 27).

As doenças, as perdas, o isolamento social, o declínio funcional, a redução da renda, a falta de infraestrutura e diversos outros fatores são os responsáveis por essa ideia pessimista a respeito do envelhecer, essas pessoas tomam atitudes e posições que não são saudáveis e não 
aceitam com tranquilidade as sucessivas mudanças desta fase da vida. Observam-se estas afirmações nas falas a seguir:

Depois que eu envelheci só sinto cansaço e dor, então envelhecer é isso (Idoso 26).

O tempo passa tem que ficar velho, trabalhei, trabalhei pra me aposentar e não ter nada (Idoso 28).

É preocupação de ficar doente e não ter quem cuide ficar nas mãos de outra pessoa (Idoso 31).

Nos respectivos sujeitos do estudo o impacto dessas mudanças é ainda mais severos devido a todos os agravos a que eles são submetidos na comunidade.

\section{DISCUSSÃO}

Para envelhecer com qualidade é necessário que se tenha uma vida ativa, produtiva, participativa e afetiva, com a família e sociedade. É fundamental que o idoso desperte a curiosidade de buscar novos conhecimentos para aperfeiçoá-los, proporcionando o prolongamento do seu autocuidado (Silva, Fossatti, \& Portella, 2007).

Diante dessa realidade, é conhecido que a dependência e falta de autonomia podem ser experienciadas de diferentes formas ao longo do ciclo vital. No processo de envelhecimento, a falta de autonomia e a dependência são comumente eventos negativos e estressantes, que conduzem à baixa $\mathrm{QV}$, tanto para quem vivencia essa condição como para aqueles que estão no seu entorno (Gonçalves et al., 2013).

Daí a importância da manutenção da autonomia do idoso, autossatisfação, participação e a possibilidade de atuar em variados contextos sociais, que pode ser alcançado mediante a existência de uma rede prestadora de serviços que contemplem tais assistências, a fim de adicionar qualidade aos anos vividos a esta população (Silva et al., 2007).
O posicionamento teórico dos entrevistados é claramente apreciado pela literatura. No decurso da existência, poucas são as pessoas que vão se instrumentalizando para lidar com as perdas que emergem com o envelhecimento. Assim, com o avanço da idade, a aquisição destes meios é vital para uma vida mais tranquila, com menos exposição ao estresse do medo de envelhecer (Farber, 2012).

Envelhecer bem é aceitar a velhice como um bem. Para atingir a sabedoria e a serenidade e para inventar nova maneira de viver, é preciso ter sido capaz de adaptar-se ao longo da vida. Na medida em que se soube viver, também se deve saber e ser capaz de envelhecer (Duarte, 2001).

Não se pode deixar de notar o máximo esforço e superação que essas pessoas devem fazer para assim refletir sobre a velhice e todo o processo de envelhecimento, já que os meios e recursos de vida disponíveis lhes são extremamente limitados e restritos e elas foram capazes de englobar ao significado de envelhecer amadurecimento e sabedoria.

Caracterizar uma pessoa como velha levando apenas a idade em consideração é medir a velhice apenas por critério cronológico, é esquecer as experiências acumuladas, os anos vividos. O tempo cronológico pode não fazer jus à jovialidade interior que mantém ativo um indivíduo de idade avançada, predisposições individuais, como relacionamento familiar, temperamento, tipo de trabalho e condições de moradia, interagem e tornam variável o conceito de envelhecimento, podendo fazer com que o indivíduo pareça mais velho do que realmente é assim como as condições favoráveis de vida podem propiciar que algumas pessoas envelheçam com aparência jovem.

Em um estudo etnográfico realizado com 
idosos do Morro da Penha, município de Santos, São Paulo, inferiu-se que os idosos indicaram que a velhice não é vista, mas sim sentida. Identificaram-se como velhos os idosos com incapacidades para realizar as atividades cotidianas e aqueles com impossibilidade de exercer seus papéis sociais, como trabalho e auxílio à família (Assis \& Martin, 2010).

Seria bastante gratificante se todos os anciãos pensassem em envelhecimento como fase de crescimento ou amadurecimento, momento pleno do ciclo vital. Porém, apesar de estar analisando discursos, cita-se que para aproximadamente $82,8 \%$ da amostra, a velhice se caracteriza como a fase mais difícil da vida, verdadeiro fardo, momento de solidão e desengano.

Percebe-se que existe um desafio a ser enfrentado e superado por essas pessoas, que é equilibrar a vontade de fazer algo que antes conseguiam fazer sem obstáculos com as atuais limitações físicas, vistas como perdas associadas ao processo de envelhecimento.

Nota-se, que as dificuldades físicas acabam por totalizar toda a experiência de envelhecer. Experimentar e salientar o processo de envelhecer como sinônimo exclusivo de doença e perdas funcionais/cognitivas reforça o medo que muitas pessoas têm de envelhecer, e assim, de adoecer e ficar dependente, o que remete à noção de finitude do ser humano (Fratezi, Silva, Silveira, \& Lopes, 2011).

Ademais, autores demonstram que o aumento da longevidade expõe a pessoa idosa a viver com enfermidades crônicas por um longo período, o que pode comprometer sua qualidade de vida. Essa condição exige políticas públicas de atenção que possibilitem a oferta de cuidados continuados e integrados às pessoas idosas no intuito de preservar a capacidade funcional e a autonomia desses sujeitos
(Gonçalves, Alvarez, Sena, Santana, \& Vicente, 2006; Gonçalves et al., 2013).

O processo de envelhecimento delimita mudanças expressivas de ordem individual, familiar e social, cada um com seus significados e relevâncias. Ao envelhecer, o idoso e sua família mudam, adquirindo determinados direitos legais e perdendo outros pelas dificuldades orgânicas e mentais trazidas pelo envelhecimento. Para muitos indivíduos enfrentar essas alterações que esse processo traz além de não ser simples, os torna mais vulneráveis a sentimentos negativos e preocupantes.

Infere-se assim que a percepção do envelhecimento é multidimensional em natureza, pois englobam as características negativas e positivas desse processo e refletem uma combinação entre precisas representações de mudanças relacionadas à idade e visões distorcidas de pessoas mais velhas (Lockenhoff et al., 2009).

\section{CONSIDERAÇÕES FINAIS}

Considera-se a velhice como processo inelutável, caracterizado por conjunto complexo de fatores fisiológicos, psicológicos e sociais de cada indivíduo. Assim certos idosos estão mais envelhecidos, outros parecem mais jovens e há ainda os que sentem não ter qualquer utilidade, afirmando a complexa heterogeneidade da velhice.

Os resultados do estudo possibilitaram traçar um perfil dos participantes, bem como das necessidades referenciadas pelos pesquisados e as maiores lacunas encontradas dizem respeito à saúde, a interação e a inclusão social.

Destarte, há de se buscar alternativas para integrar melhor esses idosos entre si e nos serviços de saúde. Uma das perspectivas é a formação de grupos voltados para problemas de saúde, como por exemplo: grupos de diabéticos e grupos de hipertensos, grupos de 
ocupação com bordados, danças, artesanatos, entre outros. Todos esses grupos possibilitam promover a saúde do idoso, integrando-o na sociedade e estimulando a convivência, interação social, fazer novas amizades, e assim, construir melhor QV.

A ESF e a enfermagem, dentro desse cenário ocupam lugar de destaque, pois têm por base à vigilância à saúde, onde o idoso é objeto precípuo de atenção. Cabendo aos mesmos o dever e o desafio de idealizarem estratégias para que se promova boa saúde e independência funcional além de serviços comunitários de apoio.

Assim, o trabalho com os idosos tem que ser prioridade, o cuidado ideal das pessoas idosas pode ser mais bem prestado por meio de um esforço de cooperação sendo necessário trabalhar na busca de melhor entendimento do processo de envelhecimento juntamente com a família proporcionando, consequentemente, um envelhecimento com QV.

Porque a verdade é que não temos de nos preocupar em viver longos anos, mas em vivêlos satisfatoriamente, viver o domínio de si mesmo depende da alma. A vida é longa quando é plena; e se faz plena quando a alma recuperou a posse de seu próprio bem e transferiu para si o domínio de si mesma.

\section{REFERÊNCIAS}

- Almeida, P. M., Mochel, E. G., \& Oliveira, M. S. S. (2011). O idoso pelo próprio idoso: percepção de si e de sua qualidade de vida. Revista Kairós Gerontologia, 13(2), 99-113. Recuperado de http://revistas.pucsp.br/index. php/kairos/article/view/5369/3849

- Andrade, F. M., et al. (2011). As características do cuidar em gerontologia na ótica da equipe multiprofissional do centro de referência à assistência social do idoso (CRASI) do município de Montes Claros (MG), Brasil.
Revista Kairós Gerontologia, 14(6), 53-71. Recuperado de http://revistas.pucsp.br/index.php/kairos/article/ view/11697/8421

- Assis, V. F. G., \& Martin, D. (2010). Falas sobre a velhice: entre o perceber e o ser idoso. Terceira Idade, 21(48), 54-65.

- Banza, M. M. S., \& José, H. M. G. (2012). Community health intervention project active ageing - ripen into health. Revista de enfermagem UFPE on line, 6(8), 18121818. Retrieved from http://www.revista.ufpe.br/revistaenfermagem/index.php/revista/article/view/3023/ pdf_1360

- Combinato, D. S., et al. (2010). Grupos de Conversa: saúde da pessoa idosa na estratégia da saúde da família. Psicologia \& Sociedade, 22(3), 558-568. Recuperado de http://www.scielo.br/pdf/psoc/v22n3/v22n3a16.pdf

- Duarte, I. A. O. (2001). O processo de envelhecimento e a assistência à saúde do idoso. Manual de enfermagem - Programa de Saúde da Família. São Paulo (SP): Ministério da Saúde/USP.

- Fabrício, S. C. C., \& Rodrigues, R. A. P. (2008). Revisão de literatura sobe fragilidade e sua relação com o envelhecimento. Revista da Rede de Enfermagem do Nordeste, 9(2), 113-119. Recuperado de http://www.revistarene. ufc.br/revista/index.php/revista/article/view/569/pdf

- Farber, S. S. (2012). Envelhecimento e elaboração das perdas. A terceira idade, estudos sobre envelhecimento, 22(53), 7-17.

- Fechine, B. R. A., \& Trompieri, N. (2012). O processo de envelhecimento: as principais alterações que acontecem com o idoso com o passar dos anos. InterSciencePlace: revista cientifica internacional, 1(7), 106-194. Recuperado de http://www.interscienceplace.org/isp/index.php/ isp/article/view/196/194

- Floriano, P. J., \& Dalgalarrondo, P. (2007). Saúde mental, qualidade de vida e religião em idosos de um programa de saúde da família, Jornal brasileiro de psiquiatria, 56(3), 162-170. Recuperado de http://www.scielo.br/ pdf/jbpsiq/v56n3/a02v56n3.pdf

- Fratezi, F. R., Silva, H. S., Silveira, M. H., \& Lopes, A. (2011). Equipamentos de assistência social de atendimento ao idoso do município de São Paulo: percepções 
sobre envelhecimento, velhice, gerontologia e o papel do gerontólogo. RBCEH, 8(1), 107-119.

- Gonçalves, L. H. T., Alvarez, A. M., Sena, E. L. S., Santana, L. W. S., \& Vicente, F. R. (2006). Perfil da família cuidadora de idoso doente/ fragilizado do contexto sociocultural de Florianópolis, SC. Texto \& Contexto Enfermagem, 15(4), 570-577.

- Gonçalves, L. T. H., et al. (2013). Convívio e cuidado familiar na quarta idade: qualidade de vida de idosos e seus cuidadores. Revista Brasileira de Geriatria e Gerontologia, 6(2), 315-325. Recuperado de http://www.scielo. br/pdf/rbgg/v16n2/11.pdf

- Jobin, E. F. C., Sousa, V. O., \& Cabrera, M. A. S. (2010). Causas de hospitalização de idosos em dois hospitais gerais pelo Sistema Único de Saúde (SUS). Acta scientiarum. Health Science, 32(1), 79-83. Recuperado de http:// periodicos.uem.br/ojs/index.php/ActaSciHealthSci/article/view/5631/5631

- Keinert, T. M. M., \& Rosa, T. E. C. (2009). Direitos humanos, envelhecimento ativo e saúde da pessoa idosa: marco legal e institucional. Envelhecimento e Saúde, (47), 4-8. Recuperado de http://periodicos.ses.sp.bvs.br/pdf/ bis/n47/a01_bisn47.pdf

- Lockenhoff, C. E., et al. (2009). Perceptions of aging across 26 cultures and their culture-level associates. Psychology and Aging, 24(4), 941-954. Retrieved from http://www.ncbi.nlm.nih.gov/pmc/articles/ PMC2933107/pdf/nihms-215302.pdf

- Marin, M. J. S., Cecílio, L. C. O., Rodrigues, L. C. R., Ricci, F. A., \& Druzian, S. (2008). Diagnósticos de enfermagem de idosas carentes de um programa de saúde da família (PSF). Escola Anna Nery Revista de Enfermagem, 12(2), 278-284. Recuperado de http://www.scielo.br/ pdf/ean/v12n2/v12n2a12

- Moliterno, A. C. M., Faller, J. W., Borghi, A. C., Marcon, S. S., \& Carreira, L. (2012). Viver em família e qualidade de vida de idosos da universidade aberta da terceira idade. Revista enfermagem UERJ, 20(2), 179-184. Recuperado de http://www.e-publicacoes.uerj.br/index.php/ enfermagemuerj/article/view/4040/2802

- Oliveira, D. C. (2008). Análise de conteúdo temáticocategorial: uma proposta de sistematização. Revista en- fermagem UERJ, 16(4), 569-576. Recuperado de http:// www.facenf.uerj.br/v16n4/v16n4a19.pdf

- Queiroz, A. A. R., Fernandes, F. M., Cirino, I. D., Oliveira, S. X., \& Menezes, R. M. P. (2010). Intervenções de enfermagem geriátrica ao idoso fragilizado [Edição especial], FIEP Bulletin, 80. Recuperado de http://www.fiepbulletin.net/index.php/fiepbulletin/article/view/1636/3185

- Rocha, F. C. V., Carvalho, C. M. R. G., Figueiredo, M. L. F., \& Caldas, C. P. (2011). O cuidado do enfermeiro ao idoso na estratégia saúde da família. Revista enfermagem UERJ, 19(2), 186-191. Recuperado de http://www.facenf.uerj.br/v19n2/v19n2a03.pdf

- Silva, C. A., Fossatti, A. F., \& Portella, M. R. (2007). Percepção do homem idoso em relação às transformações decorrentes do processo do envelhecimento humano. Estudos interdisciplinares sobre o envelhecimento, 12, 111-126.

- Tavares, D. M. S., Araújo, M. O., \& Dias, F. A. (2011). Qualidade de vida dos idosos: comparação entre distritos sanitários de Uberaba - MG. Ciência, cuidado $e$ saúde, 10(1), 74-81. Recuperado de http://www.periodicos.uem.br/ojs/index.php/CiencCuidSaude/article/ view/9897/pdf

- Teixeira, I. N. A., \& Neri, A. L. (2008). Envelhecimento bem sucedido: uma meta no curso da vida. Psicologia USP, 19(1), 81-94. Recuperado de http://www.scielo.br/ pdf/pusp/v19n1/v19n1a10.pdf

Torres, G. V., et al. (2009). Funcionalidade familiar de idosos dependentes residentes em domicílios. Avaliação Psicológica, 8(3), 415-423. Recuperado de http://pepsic. bvsalud.org/pdf/avp/v8n3/v8n3a13.pdf 